\title{
FREE BOUNDARY PROBLEMS IN CONTROLLED RELEASE PHARMACEUTICALS: II. SWELLING-CONTROLLED RELEASE*
}

\author{
DONALD S. COHEN $\dagger$ AND THOMAS ERNEUX
}

\begin{abstract}
A problem in controlled release pharmaceutical systems is formulated and studied. The device modeled is a polymer matrix containing an initially immobilized drug. The release of the drug is achieved by countercurrent diffusion through a penetrant solvent with the release rate being determined by the rate of diffusion of the solvent in the polymer. The mathematical theory yields a free boundary problem which is studied in various asymptotic regimes.
\end{abstract}

Key words. free boundaries, perturbations, polymers, controlled release

AMS(MOS) subject classifications. 35B25, 35F99, 35K20, 85A51

1. Introduction. When a glassy polymer is placed in contact with a penetrant solvent, a glassy-rubbery interface appears and moves through the polymer. For some polymer-penetrant systems, the interface velocity is constant for small times $T$ (zero order or Case II diffusion) but may vary as $T^{-1 / 2}$ at larger times (Fickian diffusion). The diffusive transport of the solvents for the various systems are modeled mathematically as moving boundary problems which are difficult to solve. In a previous paper [10] we analyzed two different types of controlled release pharmaceutical systems in which a drug is released in proportion to the rate of transport of a penetrant solvent into a glassy polymer. In both cases we found that the penetrant front moves initially at a constant rate, and our asymptotic analysis allowed us to determine the optimal conditions on the parameters for zero order release.

In the present paper we formulate and analyze the following more complex controlled release pharmaceutical system: A drug or bioactive agent is initially immobilized in a glassy polymer. The release of the drug then results from the diffusion of the dissolved drug under the countercurrent diffusion of a solvent (e.g., water or a biological fluid) into the polymer. However, it is the penetrating dissolution medium that is the controlling factor in the swellable polymeric system. To analyze mathematically the swelling-controlled release of the drug, we propose combining the Higuchi model [3a], [3b] for the transport of the drug and the Astarita and Sarti model [4] for the transport of the solvent. For the part of the model involving the latter case, our previous study [10] of specific polymer-penetrant systems will be relevant. Our combined model is similar to the problem studied by Peppas et al. [5] except for the conditions at the moving fronts.

In $\S 2$, we reexamine the Higuchi model for the release of drugs in matrix devices. In these devices, the surrounding fluid plays a minor role. It is the dissolution of the drug which is the rate-limiting step in the release process. We show that the steady state approximation proposed by Higuchi represents the leading term of an asymptotic

* Received by the editors September 10, 1986; accepted for publication (in revised form) September 16, 1987.

$\dagger$ Department of Applied Mathematics, California Institute of Technology, Pasadena, California 91125. This work was supported in part by U.S. Army Research Office (Durham) under contract DAAG29-85-K-0092, by the National Science Foundation under grant DMS-8706642, and by the Department of Energy under grant 7405/ENG-36 at the Center for Nonlinear Studies at Los Alamos.

$\ddagger$ Department of Engineering Sciences and Applied Mathematics, Northwestern University, Evanston, Illinois 60208. This work was supported in part by the Air Force Office of Scientific Research under grant AFOSR85-0150 and the National Science Foundation under grant DMS-8701302. 
expansion of the solution. This expansion will then be used in $\S 3$, when we describe the diffusion of the drug and the penetrant in swellable polymers.

2. Matrix-controlled release [6]. Matrix devices may contain the drug at an initial loading $A_{0}$ which is significantly higher than the drug solubility in the matrix. In contact with a dissolution medium, the drug diffuses through the polymer and the dissolution of the solute may be the rate-limiting step of the release process. A popular model describing this case was proposed by Higuchi in 1961 [3a]. The model has been supported by many kinetic studies and improved in several ways [7], [8]. A schematic diagram of drug release from a slab is shown in Fig. 1. At time 0 , the matrix device $\left(-X_{0}<X<X_{0}\right)$ contains dispersed drug and is in contact with the dissolution medium $\left(X<-X_{0}\right.$ and $X>X_{0}$ ). At time $T$, the drug dissolves from the surface layer of the device. An interface between dissolved and dispersed drug then moves into the interior. $\boldsymbol{A}_{s}$ is defined as the solute solubility of the drug in the polymer. The polymer does not swell during the release process and the release medium is a perfect sink. The diffusion of the solute through the dissolved matrix phase is then described by the following equations:

$$
\begin{aligned}
& A_{T}=D_{d} A_{X X}, \quad S(T)<X<X_{0}, \\
& A=0 \quad \text { at } X=X_{0}, \\
& A=A_{s} \\
& -D_{d} A_{X}=\left(A_{s}-A_{0}\right) S^{\prime}
\end{aligned}
$$

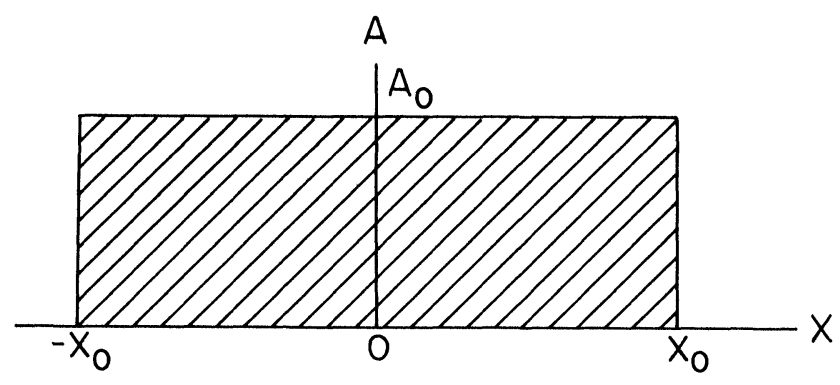

Time 0

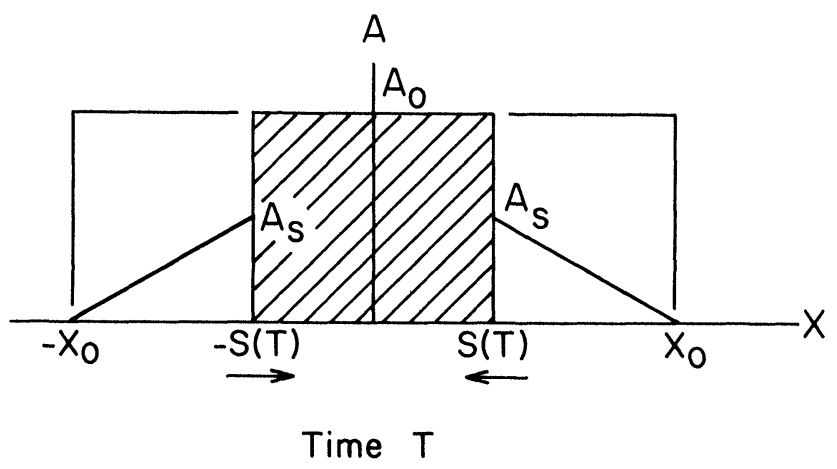

FIG. 1. Matrix-controlled release. The concentration of the drug $A$ is represented as a function of $X$. The cross-hatched region corresponds to the region of the matrix where the drug is dispersed and has not been dissolved. 


$$
S(0)=X_{0}, \quad S(T)>0 .
$$

The boundary condition (2.2) describes the perfect sink of the drug, (2.4) is a mass balance equation at the moving front, and $S^{\prime}=d S / d T$. Equation (2.3) means that the drug solubility controls the kinetics of the interface. By defining the new variables

$$
u=\frac{A}{A_{s}}, \quad t=\frac{T D_{d} A_{s}}{\left(A_{0} X_{0}^{2}\right)}, \quad x=\frac{X}{X_{0}}, \quad L(t)=\frac{S(T)}{X_{0}},
$$

(2.1)-(2.5) can be rewritten in a simpler way:

$$
\begin{aligned}
& u_{t}=\eta^{-1} u_{x x}, \quad L(t)<x<1, \\
& u=0 \quad \text { at } x=1, \\
& u=1 \quad \text { at } x=L(t), \\
& -u_{x}=(\eta-1) L^{\prime} \\
& L(0)=1, \quad L(t)>0,
\end{aligned}
$$

where $L^{\prime}=d L / d t$ and $\eta$ is defined by

$$
\eta=\frac{A_{s}}{A_{0}} \ll 1 .
$$

It is mathematically convenient to introduce a new spatial variable defined by

$$
y=\frac{1-x}{1-L(t)}
$$

and to reformulate (2.7)-(2.11) in terms of $y$ and $t$ :

$$
\begin{aligned}
& u_{y y}=\eta\left[(1-L)^{2} u_{t}+L^{\prime}(1-L) y u_{y}\right], \quad 0<y<1, \\
& u=0 \quad \text { at } y=0, \\
& u=1 \\
& u_{y}=(1-L)(\eta-1) L^{\prime} \quad \text { at } y=1, \\
& L(0)=1, \quad L(t)>0 .
\end{aligned}
$$

We then seek a solution of these equations of the form

$$
\begin{aligned}
& u(y, t, \eta)=u_{0}(y, t)+\eta u_{1}(y, t)+\cdots, \\
& L(t, \eta)=L_{0}(t)+\eta L_{1}(t)+\cdots .
\end{aligned}
$$

From the leading order problem, we find $u_{0}=y$ and $L_{0}=1-(2 t)^{1 / 2}$. Thus, we conclude that

$$
A(X, T)=A_{s}\left(\frac{X-X_{0}}{S(T)-X_{0}}\right)+O\left(\eta A_{s}\right)
$$

and

$$
S(T)=X_{0}-\left(2 T D_{d} A_{s} / A_{0}\right)^{1 / 2}+O\left(\eta X_{0}\right) .
$$

Moreover, if the release rate is defined by

$$
M=S_{u}\left[-D_{d} A_{X}\right]_{X=X_{0}},
$$


where $S_{u}$ is the area of solute diffusion, we obtain using (2.21) and (2.22)

$$
M \sim S_{u}\left[D_{d} A_{s} A_{0} /(2 T)\right]^{1 / 2},
$$

which is exactly the Higuchi result using a pseudo-steady-state approximation [6]. Note from (2.22) that the position of the front varies like $(2 T)^{1 / 2}$ for all time and is typical of matrix-controlled releases. Observations of constant release for small time have motivated modifications of the Higuchi model (partition controlled release [7]). We now explore the advantage of swelling-controlled release by supplementing the Higuchi model with a model for the solvent diffusion through the swellable polymer.

3. Swelling-controlled release [1], [2]. In these systems a drug is dissolved or dispersed within a polymer matrix and is not able to diffuse through the matrix. In contact with environmental fluid, the matrix starts to swell and the drug contained in that part of the polymer diffuses through the polymer. Thus the release rate is determined by the rate of diffusion of the solvent in the polymer. A schematic diagram of the concentration profile in a slab matrix for the drug (full line) and the solvent (broken line) is shown in Fig. 2. At time 0, the matrix is exposed to a penetrant that causes swelling of the glassy polymer. The concentrations of the solute and the penetrant are denoted by $A$ and $C$, respectively. $C_{0}$ is the solubility of the solvent in the swollen polymer. Two moving fronts are observed. The front located at $X=S_{1}(T)$ separates the solvent-free polymer from the swollen polymer; the front located at $X=S_{2}(T)$

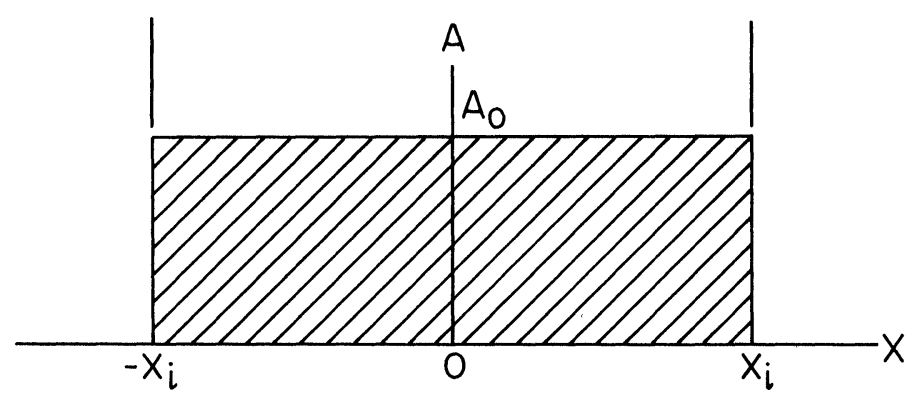

Time 0

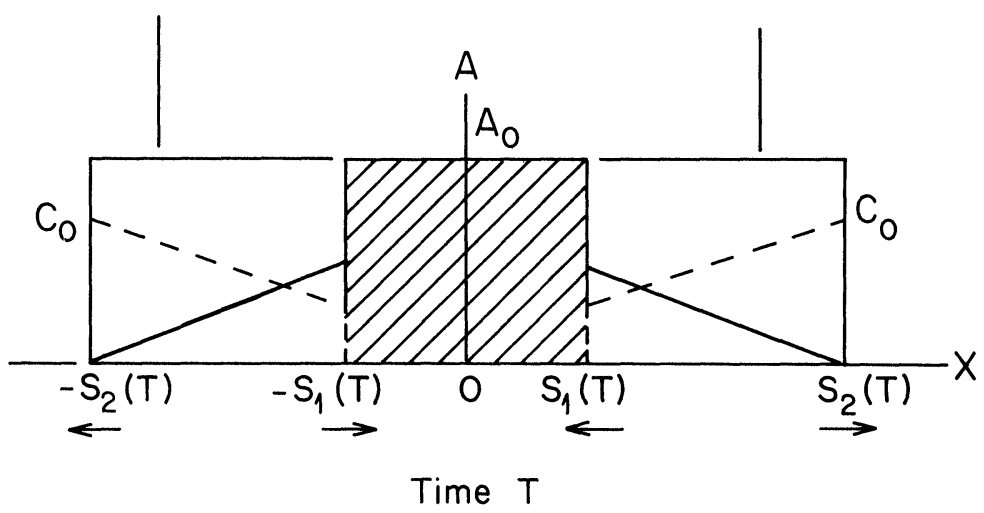

FIG. 2. Swelling-controlled release. The concentration of the drug $A$ (full line) and the concentration of the solvent $C$ (broken line) are represented as functions of $X$. 
results from the volume expansion of the polymer due to the gradual swelling. As in [5], we assume that $S_{2}(T)$ can be obtained by the following equation

$$
S_{2}(T)-X_{i}=\bar{v} \int_{S_{1}(T)}^{S_{2}(T)} C\left(T, X^{\prime}\right) d X^{\prime}
$$

where $X=X_{i}$ is the initial position of the front and $\bar{v}$ is the molar volume of the swelling agent. Using the Higuchi model, we describe the diffusion of the drug by

$$
\begin{gathered}
A_{T}=D_{d} A_{X X}, \quad S_{1}(T)<X<S_{2}(T), \\
A=0 \quad \text { at } X=S_{2}(T), \\
-D_{d} A_{X}=\left(A-A_{0}\right) S_{1}^{\prime} \quad \text { at } X=S_{1}(T), \\
S_{1}(0)=S_{2}(0)=X_{i} .
\end{gathered}
$$

These equations are equivalent to (2.1), (2.2), and (2.4). Equation (2.3) is not required because we now assume that it is the diffusion of the swelling agent that controls the rate of the release. We model its transport by the equations previously investigated [10]. They are given by

$$
\begin{gathered}
C_{T}=D C_{X X}, \quad S_{1}(T)<X<S_{2}(T), \\
C=C_{0} \quad \text { at } X=S_{2}(T), \\
-D C_{X}=(C+K) S_{1}^{\prime} \quad \text { at } X=S_{1}(T), \\
S_{1}^{\prime}=-k_{1}\left(C-C^{*}\right)^{n} \quad \text { at } X=S_{1}(T),
\end{gathered}
$$

$k_{1}, K, D, C_{0}$ and $C^{*}$ given are constant parameters. Equation (3.6) is Fick's Diffusion Law for a one-dimensional system subject to a fixed concentration at $X=S_{2}(T)$ (3.7). Equation (3.9) describes the swelling kinetics as an interface reaction: the excess $C(X, T)-C^{*}$ over the equilibrium value $C^{*}$ drives the penetrant front at some finite rate. In this equation $k_{1}$ and $n$ represent phenomenological quantities. Equation (3.8) is a mass balance at the moving front. It is derived in the following way: the flux from the swelling region across $X=S_{1}(T)$ is given by $D C_{X}+C S_{1}^{\prime}$ and is assumed to be proportional to the flux generated by the interface region. Thus we have

$$
D C_{X}+C S_{1}^{\prime}=k_{2}\left(C-C^{*}\right)^{n} \quad \text { at } X=S_{1}(T),
$$

and using (3.9), we obtain (3.8) with $K=k_{2} / k_{1}$.

Our model for the diffusion of the drug and the solvent is similar to the problem formulated by Peppas et al. [5]. It mainly differs from our formulation by the equations at the moving front. In its one-phase formulation $\left(\varepsilon=D_{1} / D_{2}=0\right.$ in [5]), the model proposed by Peppas et al. assumed $C_{X}=0, C=C^{*}$, and $A_{X}=0$ at $X=S_{1}(T)$. These conditions can be obtained from (3.4), (3.8), and (3.9) provided that $D_{d}, D$, and $k_{1}$ are sufficiently large. In order to analyze (3.1)-(3.9), we first introduce the dimensionless variables $u, v, t, x, L_{1}$, and $L_{2}$ defined by

$$
\begin{aligned}
& u=\frac{C-C^{*}}{C_{0}-C^{*}}, \quad v=\frac{A}{A_{0}}, \quad t=\frac{T}{\alpha}, \\
& x=\frac{X}{\beta}, \quad L_{i}=\frac{X_{i}}{\beta}, \quad L_{j}(t)=\frac{S_{j}(T)}{\beta} \quad(j=1 \text { or } 2)
\end{aligned}
$$

where

$$
\alpha=\frac{D\left(C_{0}-C^{*}\right)^{1-2 n}}{\bar{C} k_{1}^{2}}, \quad \beta=\frac{D\left(C_{0}-C^{*}\right)^{1-n}}{\bar{C} k_{1}}
$$


and

(3.10d)

$$
\bar{C}=C^{*}+K
$$

Then (3.1)-(3.9) become:

$$
\begin{gathered}
u_{t}=\varepsilon^{-1} u_{x x}, \quad L_{1}(t)<x<L_{2}(t), \\
u=1 \quad \text { at } x=L_{2}(t), \\
-u_{x}=(1+\varepsilon u) L_{1}^{\prime} \quad \text { at } x=L_{1}(t), \\
L_{1}^{\prime}=-u^{n} \quad \text { at } x=L_{1}(t), \\
L_{2}(t)-L_{i}=\bar{v} \bar{C} \int_{L_{1}(t)}^{L_{2}(t)}\left[\frac{C^{*}}{\bar{C}}+\varepsilon u\right] d x, \\
v_{t}=\frac{D_{d}}{D} \varepsilon^{-1} v_{x x}, \quad L_{1}(t)<x<L_{2}(t), \\
v=0 \quad \text { at } x=L_{2}(t), \\
-\frac{D_{d}}{D} v_{x}=\varepsilon(v-1) L_{1}^{\prime} \quad \text { at } x=L_{1}(t), \\
L_{1}(0)=L_{2}(0)=L_{i}
\end{gathered}
$$

where $\varepsilon$ is a control parameter defined by

$$
\varepsilon=\frac{C_{0}-C^{*}}{\bar{C}}>0
$$

The results of our previous study [10] indicate that zero-order release can be achieved for a long period of time if $\varepsilon$ is sufficiently small. This suggests analyzing (3.11)-(3.19) in the limit $\varepsilon \rightarrow 0$. To this end, we seek a solution of these equations of the form

$$
\begin{gathered}
u(t, y, \varepsilon)=u_{0}(t, y)+\varepsilon u_{1}(t, y)+\cdots, \\
v(t, y, \varepsilon)+v_{0}(t, y)+\varepsilon v_{1}(t, y)+\cdots
\end{gathered}
$$

where

$$
y=\frac{x-L_{1}}{L_{2}-L_{1}}
$$

and

$$
L_{j}(t, \varepsilon)=L_{j 0}(t)+\varepsilon L_{j 1}(t)+\cdots \quad(j=1 \text { or } 2) .
$$

After introducing (3.21)-(3.24) into (3.12)-(3.20) and equating to zero the coefficients of each power of $\varepsilon$, we obtain the following problems for $u_{0}$ and $v_{0}$ :

$$
\begin{gathered}
u_{0 y y}=v_{0 y y}=0, \quad 0<y<1, \\
u_{0}-1=v_{0}=0 \quad \text { at } y=1, \\
-u_{0 y}=\left(L_{20}-L_{10}\right) L_{10}^{\prime}, \quad v_{0 y}=0 \quad \text { at } y=0, \\
L_{10}^{\prime}=-u_{0}^{n} \quad \text { at } y=0, \\
L_{20}(t)-L_{i}=\bar{v} C^{*}\left[L_{20}(t)-L_{10}(t)\right] \\
L_{10}(0)=L_{20}(0)=L_{i} .
\end{gathered}
$$


These equations are easily solved. We find that

$$
u_{0}(y, t)=F(t)(y-1)+1, \quad v_{0}(y, t)=0
$$

where $F(t)$ satisfies the conditions

$$
\begin{gathered}
-F=\left(L_{20}-L_{10}\right) L_{10}^{\prime}, \\
L_{10}^{\prime}=-(1-F)^{n}
\end{gathered}
$$

and

$$
L_{20}(t)=\frac{L_{i}-\bar{v} C^{*} L_{10}(t)}{\left(1-\bar{v} C^{*}\right)} .
$$

Using (3.34), we can eliminate $L_{20}(t)$ in (3.32) and (3.33):

$$
\begin{gathered}
-F\left(1-\bar{v} C^{*}\right)=\left(L_{i}-L_{10}\right) L_{10}^{\prime}, \\
L_{10}^{\prime}=-(1-F)^{n} .
\end{gathered}
$$

By defining $L_{0}=L_{i}-L_{10}$, the problem (3.35)-(3.36) becomes similar to (2.49)-(2.50) in $[10]$

$$
\begin{gathered}
F\left(1-\bar{v} C^{*}\right)=L_{0} L_{0}^{\prime}, \\
L_{0}^{\prime}=(1-F)^{n} .
\end{gathered}
$$

Eliminating $L_{0}^{\prime}$, we obtain $L_{0}=L_{0}(F(t))$

$$
L_{0}(F(t))=\frac{F\left(1-\bar{v} C^{*}\right)}{(1-F)^{n}}
$$

where $F \geqq 0$. Then using (3.39) and (3.38), we obtain a differential equation for $F(t)$ which can be integrated. As a simple illustration, we consider the case $n=1$. Using (3.39), (3.38) is easily solved. We find that

$$
L_{0}=L_{i}-L_{10}(t)=-\Gamma+\left(\Gamma^{2}+2 \Gamma t\right)^{1 / 2}
$$

where $\Gamma=\left(1-\bar{v} C^{*}\right)$. Of particular interest is the small time behavior. From (3.40), we find that

$$
L_{i}-L_{10}(t)=t+\frac{t^{2}}{2 \Gamma}+O\left(t^{3}\right)
$$

Since $v_{0}=0$, we must analyze the problem for $v_{1}(y, t)$ which is given by

$$
\begin{gathered}
v_{1 y y}=0, \quad 0<y<1, \\
v_{1}=0 \quad \text { at } y=1, \\
\frac{D_{d}}{D} v_{1 y}=\left(L_{20}-L_{10}\right) L_{10}^{\prime} \quad \text { at } y=1 .
\end{gathered}
$$

From (3.42) and (3.43), we find that

$$
v_{1}(y, t)=G(t)(y-1)
$$

where $G(t)$ is an arbitrary function of $t$ which is determined using (3.44):

$$
G(t)=\frac{D}{D_{d}}\left(L_{20}-L_{10}\right) L_{10}^{\prime}
$$


In conclusion, using the definition of $y,(3.44)$ and (3.45), we find that the concentration of the drug is given by

$$
v(x, t)=\varepsilon \frac{D}{D_{d}} L_{10}^{\prime}\left(x-L_{20}\right)+O\left(\varepsilon^{2}\right)
$$

or equivalently

$$
A(X, T) \sim A_{0} \varepsilon \frac{D}{D_{d}} L_{10}^{\prime} \beta^{-1}\left(X-S_{2}(T)\right) .
$$

With (3.48), we can determine the drug release rate $M=S_{u}\left[-D_{d} A_{X}\right]_{X=S_{2}}$. It is given by

$$
M-S_{u} A_{0}\left[S_{1}^{\prime}(0)\right] L_{10}^{\prime}
$$

where $S_{1}^{\prime}(0)$ is the initial velocity of the polymer front. It is defined by (3.9) with the initial condition $C\left(X_{i}, 0\right)=C_{0}$. Since $L_{10}^{\prime} \sim-1$ for small time (from (3.35) and (3.36), $F \sim 0$ and $L_{10}^{\prime} \sim-1$ near $\left.t=0\right)$, the drug release rate is constant initially. The expression (3.49) is in agreement with the ideas formulated by Peppas and Franson [9].

4. Discussion. In this paper, we have formulated and analyzed a mathematical model for the swelling controlled release of drugs. The model combines Higuchi equations [3a], [3b] and Astarita and Sarti equations [4] for the diffusion of the drug and the solvent, respectively.

In $\S 2$, we show that Higuchi's approximation (the so-called pseudo-steady-state approximation) represents the first term of an asymptotic expansion. The small parameter used in this expansion is $\eta=A_{s} / A_{0}$ where $A_{s}$ and $A_{0}$ are the solute solubility and the solute initial loading, respectively. Although Higuchi equations can be solved exactly [11] and better approximative solutions have been proposed [12], our asymptotic approach is a systematic method to obtain higher order corrections. Because of its simplicity, it can also be used to analyze more complex moving boundary problems.

In the second part of the paper, we proposed a similar asymptotic method to analyze our model for the diffusion of the drug and the solvent. The small parameter is now given by $\varepsilon$ which is proportional to the deviation $C_{0}-C^{*}$ where $C_{0}$ is the concentration of the solvent at the polymer/solvent interface and $C^{*}$ corresponds to the equilibrium value of the solvent concentration in the polymer. As in our previous analysis, the first term of our asymptotic expansion represents a quasi-steady-state approximation for both the drug and the solvent. The drug release rate is given by (3.49) and is constant for small time: $M \simeq S u A_{0} S_{1}^{\prime}(0)=S u A_{0} k_{1}\left(C_{0}-C^{*}\right)^{n}$. This constant is proportional to the deviation $C_{0}-C^{*}$. We note that the volume expansion of the polymer does not contribute to the expression of $M$ for small time.

\section{REFERENCES}

[1] R. W. Korsmeyer AND N. A. Peppas, Macromolecular and modeling aspects of swelling-controlled systems, in Controlled Release Delivery Systems, T. J. Roseman and S. Z. Mansdorf, eds., Marcel Dekker, 1983.

[2] H. B. HOPFENBERG AND K. C. Hsu, Swelling-controlled, constant rate delivery systems, Polymer Engrg. and Sci., 18 (1978), pp. 1186-1191.

[3a] T. HIGUCHI, Rate of release of medicaments from ointment bases containing drugs in suspension, J. Pharmac. Sci., 50 (1961), pp. 874-875.

[3b] - Mechanism of sustained-action medication. Theoretical analysis of rate of release of solid drugs dispersed in solid matrices, J. Pharmac. Sci., 52 (1963), pp. 1145-1149. 
[4] G. Astarita AND G. C. SARTI, A class of mathematical models for sorption of swelling solvents in glassy polymers, Polymer Engrg. and Sci., 18 (1978), pp. 338-395.

[5] N. A. PePpas ET AL., Modelling of drug diffusion through swellable polymeric systems, J. Membrane Sci., 7 (1980), pp. 241-253.

[6] N. A. PEPPAS, Mathematical models for controlled release kinetics, in Medical Applications of Controlled Release, vol. II, R. S. Langer and D. L. Wise, eds., CRC Press, 1984.

[7] T. J. RosemAN AND W. I. HiguCHI, Release of Medroxyprogesterone acetate from a silicone polymer, J. Pharmac. Sci., 59 (1970), pp. 353-357.

[8] Y. W. ChIEN AND H. J. LAMBERT, Controlled drug release from polymeric delivery devices II: differentiation between partition-controlled and matrix-controlled drug release mechanisms, J. Pharmac. Sci., 63 (1974), pp. 515-519.

[9] N. A. PEPPAS AND N. M. FRANSON, The swelling interface number as a criterion for prediction of diffusional solute release mechanisms in swellable polymers, J. Polymer Sci., 21 (1983), pp. 983-997.

[10] D. S. COHEN AND T. ERNEUX, Free boundary problems in controlled release pharmaceuticals. I: Diffusion in glassy polymers, SIAM J. Appl. Math., this issue, pp. 1451-1465.

[11] D. R. PAul AND S. K. MCSPADDEN, Diffusional release of a solute from a polymer matrix, J. Membrane Sci., 1 (1976), pp. 33-41.

[12] P. I. LEE, Diffusional release of a solute from a polymeric matrix-approximate analytical solutions, J. Membrane Sci., 7 (1980), pp. 255-275. 